\title{
Effects of unidirectional flow shear stresses on the formation, fractal microstructure and rigidity of incipient whole blood clots and fibrin gels
}

\author{
N. Badiei ${ }^{\mathrm{a}}$, A.M. Sowedan ${ }^{\mathrm{a}, \mathrm{b}}$, D.J. Curtis ${ }^{\mathrm{a}, *}$, M.R. Brown ${ }^{\mathrm{a}}$, M.J. Lawrence ${ }^{\mathrm{b}, \mathrm{c}}$, A.I. Campbell ${ }^{\mathrm{a}}$,

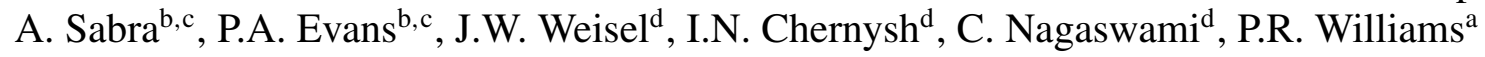 \\ and K. Hawkins ${ }^{\mathrm{b}, \mathrm{c}}$

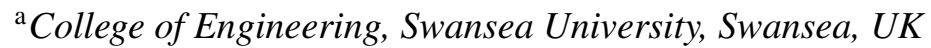 \\ ${ }^{\mathrm{b}}$ College of Medicine, Swansea University, Swansea, UK \\ ${ }^{\mathrm{c}}$ NISCHR Clinical Haemostasis and Biomarker Research Unit, ABMU NHS Trust Hospital, \\ Morriston, Swansea, UK \\ ${ }^{\mathrm{d}}$ Department of Cell and Developmental Biology, Perelman School of Medicine, University \\ of Pennsylvania, Philadelphia, PA, USA
}

\begin{abstract}
Incipient clot formation in whole blood and fibrin gels was studied by the rheometric techniques of controlled stress parallel superposition (CSPS) and small amplitude oscillatory shear (SAOS). The effects of unidirectional shear stress on incipient clot microstructure, formation kinetics and elasticity are reported in terms of the fractal dimension $\left(d_{f}\right)$ of the fibrin network, the gel network formation time $\left(T_{G P}\right)$ and the shear elastic modulus, respectively. The results of this first haemorheological application of CSPS reveal the marked sensitivity of incipient clot microstructure to physiologically relevant levels of shear stress, these being an order of magnitude lower than have previously been studied by SAOS. CSPS tests revealed that exposure of forming clots to increasing levels of shear stress produces a corresponding elevation in $d_{f}$, consistent with the formation of tighter, more compact clot microstructures under unidirectional flow. A corresponding increase in shear elasticity was recorded. The scaling relationship established between shear elasticity and $d_{f}$ for fibrin clots and whole blood confirms the fibrin network as the dominant microstructural component of the incipient clot in terms of its response to imposed stress. Supplementary studies of fibrin clot formation by rheometry and microscopy revealed the substantial additional network mass required to increase $d_{f}$ and provide evidence to support the hypothesis that microstructural changes in blood clotted under unidirectional shear may be attributed to flow enhanced thrombin generation and activation. CSPS also identified a threshold value of unidirectional shear stress above which no incipient clot formation could be detected. CSPS was shown to be a valuable haemorheological tool for the study of the effects of physiological and pathological levels of shear on clot properties.
\end{abstract}

Keywords: Gel point, clotting time, fractal dimension, shear stress, controlled stress parallel superposition

\section{Introduction}

Blood coagulation involves the formation of haemostatic plugs called clots at lesions of the circulatory system. In addition to the initial haemostatic response of platelets to subendothelial cells or foreign

\footnotetext{
${ }^{*}$ Corresponding author: Dr. Daniel J. Curtis, College of Engineering, Swansea University, Swansea, UK. Tel.: +44 01792 606473; E-mail: d.j.curtis@swansea.ac.uk. 
surfaces [20,31], the clotting process involves fibrinogen-fibrin transformation due to the catalytic action of thrombin and the subsequent polymerisation and network formation of fibrin fibres which serves to stabilise the initial platelet plug $[1,38]$. In vivo the network of fibrin fibres forms the primary microstructural basis of a clot whereas in vitro they form a fibrin gel [26].

Viscoelastic properties are among the most sensitive measures of fibrin polymerization and clot microstructure [38]. In haemorheological measurements the clotting time may be identified with that required to form a sample-spanning network of fibrin fibres at the Gel Point, GP [13, 39]. The GP marks the viscoelastic fluid to solid transition and corresponds to the establishment of the incipient clot [14]. Accurate techniques for GP detection are based on measurements of the viscoelastic properties of incipient clots [39]. A fractal analysis of such measurements quantifies incipient clot microstructure in fibrin gels and blood in terms of a fractal dimension, $d_{f}[12,14,15,22,23]$. In healthy whole blood a narrow range of $d_{f}(1.74 \pm 0.04)$ provides a Healthy Index for normal clotting [14]. Enhanced clot rigidity, higher internal connectivity of fibrin network mass and decreased permeability correspond to higher values of $d_{f}$ whereas lower values, such as those reported in anticoagulated blood, correspond to weaker gels with more open, less dense fibrin networks $[12,14,15]$.

The ability to quantify clot microstructure has clinical significance. In vitro clot microstructure correlates with epidemiological and clinical data in cardiovascular diseases [36]. Patients with thrombotic disorders form plasma clots in vitro with altered microstructure, these clots being more rigid and less permeable than those from control subjects [24]. Among the many factors that affect fibrin clot microstructure is shear flow, which modulates the kinetics of fibrin formation and polymerisation [29]. Flow has a direct effect on individual platelets, including exposure of anionic phospholipids, which provide a surface for thrombin generation [25]. The shear rates associated with physiological flows lead to activation of platelets and thrombin release, with high levels of thrombin resulting in the production of thinner fibrin fibres with greater number of branchpoints [39]. Although flow can wash away thrombin or agonists released from platelets like ADP and hence decrease clotting, it can also result in a 'denser' microstructure by bringing more fibrinogen to the forming clot [29]. Fibrin fibre orientation along the direction of flow [3, 17, 41] also has important consequences for clot mechanical properties and susceptibility to fibrinolysis $[3,37]$ and it has been proposed that shear forces of the blood stream determine the likelihood of embolization [7].

The effects of flow on the microstructure of blood clots at the earliest (incipient) stage of their formation have not previously been reported. This is a significant omission insofar as the incipient fibrin clot provides a microstructural template for ensuing clot development [12]. Flow induced changes in the assembly of the template may thus have significant consequences for clot elasticity and susceptibility to fibrinolysis and hence for clot quality [3]. The present study was undertaken to elucidate the consequences of imposing unidirectional flow shear stresses on the microstructural and mechanical properties of incipient clots, as they formed, in whole blood and fibrin gels. The rheometric technique employed was controlled stress parallel superposition, CSPS, the present work being the first to employ CSPS to study blood clot formation. Additional rheometric and microscopy studies were undertaken on samples of blood and fibrin clots.

\section{Experimental}

\subsection{Small amplitude oscillatory shear, SAOS}

A controlled stress rheometer (AR-G2, TA Instruments) was used to measure the elastic and viscous components ( $G^{\prime}$ and $G^{\prime \prime}$, respectively) of the complex shear modulus, $G^{*}$. At the GP, $G^{\prime}$ and $G^{\prime \prime}$ scale in 
oscillatory frequency, $\omega$, as $G^{\prime}(\omega) \sim G^{\prime \prime}(\omega) \sim \omega^{\alpha}$ [5]. Thus the GP is identified by attainment of frequency independence of the loss tangent, $\tan \delta$, where $\tan \delta=G^{\prime \prime} / G^{\prime}$ and $\delta$ is the phase angle between oscillatory stress and strain waveforms.

The microstructure of dilute fibrin gels, at relatively low fibrinogen concentration, has previously been reported in terms of a fractal analysis of light scattering data [16]. For more concentrated systems such as whole blood a fractal analysis of viscoelastic GP data provides a basis for quantifying the structural complexity of the incipient clot's fibrin network [12, 14, 15, 22, 23]. Prior to the GP isolated clusters of branching fibrin fibres formed from polymerized monomers grow in the sol phase as loosely coupled bundles of flexible polymers [30]. At the GP a sample-spanning gel network is established as a continuous path between the sample's bounding surfaces. The polymerizing system is macroscopically homogeneous at a length-scale $L$ greater than the correlation length $\varepsilon$, whereas for $L \ll \varepsilon$ the network cluster is a fractal object whose mass $M$ scales as $M \sim L^{d f}$, where $d_{f}$ is the fractal dimension of the network. The greater the value of $d_{f}$ the more compact is the network, whereas lower values of $d_{f}$ correspond to more open/permeable networks. The exponent $\alpha$ obtained from GP measurements is a measure of gel network branching and is related to $d_{f}$ as $d_{f}=(D+2)(2 \alpha-D) / 2(\alpha-D)$, where $D$ is the Euclidian dimension $(D=3)$ [28]. It is important to note that $d_{f}$ is inferred from the power law scaling in both the storage (elastic) and viscous (loss) moduli at the gel point, the scaling exponent $\alpha$ (and thus $d_{f}$ ) being independent of the values of the storage modulus, $G^{\prime}$, at any single frequency.

In fibrin-thrombin gels formed over a range of thrombin concentration, incipient clots are characterised by values of $d_{f}$ consistent with limiting values for fractal clusters formed by diffusion limited cluster-cluster aggregation and reaction limited cluster-cluster aggregation processes [11, 33, 40].

\subsection{Controlled stress parallel superposition, CSPS}

CSPS was used to apply combined unidirectional and oscillatory shear stresses ( $\sigma_{s}$ and $\sigma_{o}$, respectively) to samples of clotting blood and fibrin-thrombin solutions, the resulting unidirectional and oscillatory flow components being parallel to each other (see Fig. 1). It is interesting to note that a similar, though significantly more complex, superposed flow regime exists within the pulsatile flow dynamics of the circulatory system. The net accumulated strain is zero under SAOS whereas the accumulated unidirectional strain increases progressively in CSPS. In controlled deformation rate superposition, the flow is described in terms of its oscillatory and steady components. This is not appropriate to the present CSPS work, which involves a time-varying unidirectional shear rate under constant stress due to rheological changes during clotting. Thus we refer herein to the unidirectional flow component, rather than a steady component.

The shear stress in parallel superposition at low amplitude is given by:

$$
\sigma(t)=\sigma_{s}+\left|G_{\|}^{*}\right| \gamma_{0} \sin \left(\omega t+\delta_{\|}\right)
$$

where $\sigma_{s}$ is the unidirectional component of stress, $\left|G_{\|}^{*}\right|$ is termed the parallel complex modulus, $\gamma_{0}$ is the oscillatory shear strain amplitude, $\omega$ is the angular frequency and $\delta_{\|}$is the parallel superposition phase angle. The parallel storage modulus, and loss modulus, $G_{\|}^{\prime}$ and $G_{\|}^{\prime \prime}$ respectively, can be determined as follows [2]:

$$
\begin{aligned}
& G_{\|}^{\prime}=G_{\|}^{*} \cos \left(\delta_{\|}\right) \\
& G_{\|}^{\prime \prime}=G_{\|}^{*} \sin \left(\delta_{\|}\right)
\end{aligned}
$$



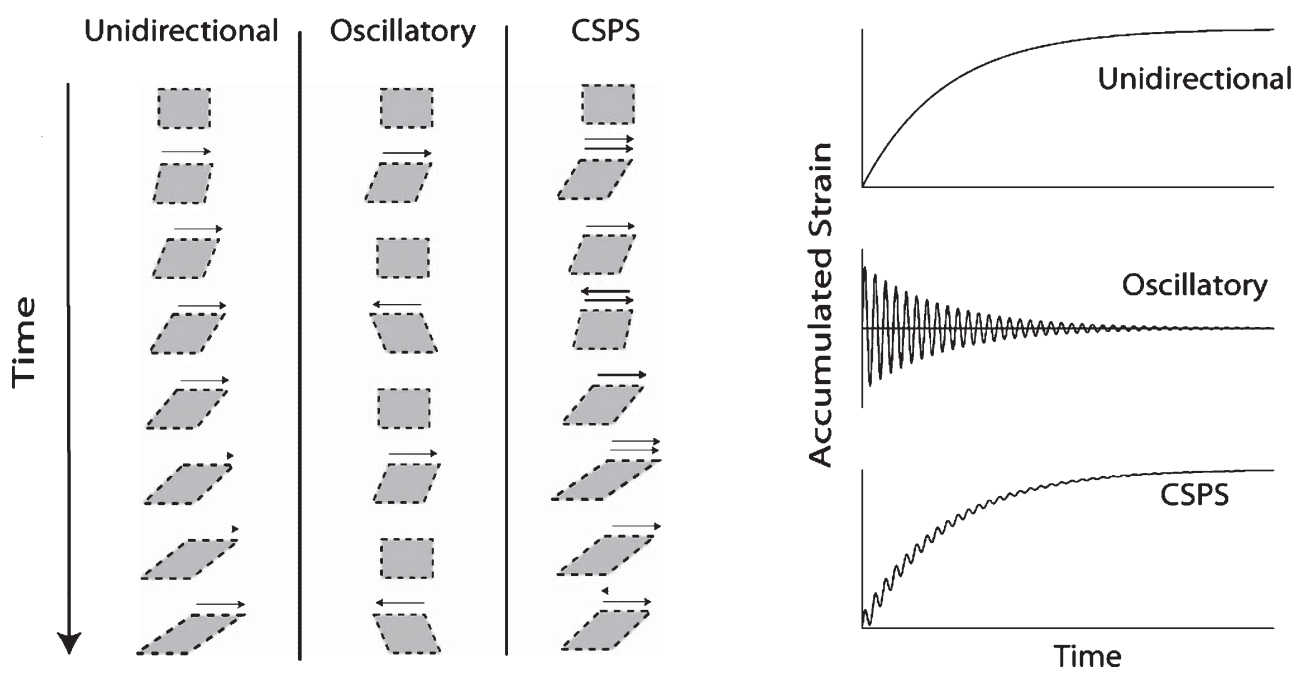

Fig. 1. Illustration of CSPS. The technique is used to apply combined unidirectional and oscillatory shear stresses $\left(\sigma_{s}\right.$ and $\sigma_{o}$, respectively), the direction of the two resulting shear motions (i.e. the unidirectional and oscillatory components) being parallel (see Fig. 1(i)). The net accumulated unidirectional strain is zero under SAOS whereas it increases progressively in CSPS (Fig. 1(ii)). The SAOS strain decreases progressively in blood as the GP is approached. CSPS involves a time-varying shear rate under constant stress due to rheological changes associated with clotting. The unidirectional flow shear rate becomes vanishingly small in the vicinity of the GP.

In the present experiments, $G_{\|}^{\prime}$ and $G_{\|}^{\prime \prime}$ were independent of oscillatory strain amplitude, allowing the oscillatory flow to probe microstructural changes. CSPS was used over a range of $\sigma_{s}$ and a range of frequencies $(0.2 \mathrm{~Hz}$ to $2 \mathrm{~Hz})$ to record the incipient clot formation time, $T_{G P}$ (the 'clotting time') and the corresponding values of $G_{\|}^{\prime}$ and $G_{\|}^{\prime \prime}$. In CSPS the unidirectional flow shear rate $\dot{\gamma}_{s}$ becomes vanishingly small in the vicinity of the GP ensuring no significant difference between $G_{\|}^{\prime}, G_{\|}^{\prime \prime}$ or $\delta_{\|}$and their corresponding parameters in SAOS [35]. Accordingly, the incipient clot's fractal dimension, $d_{f}$, was estimated using the relationship given in Section 2.1 where the value of $\alpha$ was calculated from the frequency independent value of $\delta_{\|}$recorded at the GP. Fourier Transform analysis of the 3rd harmonic component of the oscillatory strain waveforms was used to ensure that measurements were conducted within the linear viscoelastic range, as in previous SAOS studies of biopolymer gelation and blood clotting $[15,19]$.

\subsection{Blood samples}

Blood was collected from 8 healthy volunteers following informed consent and full ethical approval. All donors denied taking any medication for two weeks prior to the day of collection, on which blood was withdrawn into 5-7 Vacutainer ${ }^{\mathrm{TM}}$ tubes $(4.5 \mathrm{~mL}$; Becton \& Dickinson Co., UK) containing $3.8 \%$ sodium citrate to form a mixture of 9:1 vol./vol. blood to citrate, respectively. This was done, without any stasis, from an antecubital vein by venipuncture using a 21-gauge butterfly needle (Greiner Bio-one $\mathrm{GmbH}-$ Austria). Sodium citrate, an anticoagulant that binds the calcium ions in the blood, prevented further coagulation. Its effect was reversed by adding $0.2 \mathrm{M}$ Calcium Chloride, $\mathrm{CaCl}_{2}$ (Sigma-Aldrich) [42] to a final concentration of $0.005 \mathrm{M}$. The citrated blood was kept in a water bath at $37^{\circ} \mathrm{C}$ during the experiment and was used, in every case, within 4 hours of collection. 
SAOS and CSPS measurements were performed using an aluminium parallel plate measuring geometry $(6 \mathrm{~cm}$ diameter). In this geometry, unlike the cone and plate, the shear rate is not uniform throughout the shearing gap. However, the presence of cellular material within whole blood samples precluded the use of the latter. Samples of blood were loaded by pipette onto the temperature controlled lower plate $\left(37^{\circ} \mathrm{C}\right)$, the upper plate being immediately lowered thereafter to the preset shearing gap $(300 \mu \mathrm{m})$. A thin layer of low viscosity ( $9.4 \mathrm{mPa} . \mathrm{s})$ silicon oil prevented evaporation. The peak oscillatory stress, $\sigma_{0}$, was set at a level which ensured adequate waveform resolution and linear viscoelastic responses. The steady applied stress $\sigma_{s}$ used to generate the unidirectional flow was adjusted in each experiment.

\subsection{Fibrin-thrombin gels}

Human fibrinogen $(43.0 \mathrm{mg} / \mathrm{ml})$ and human- $\alpha$-thrombin $(500 \mathrm{NIH} / \mathrm{ml})$ were obtained from Enzyme Research Laboratories Ltd, UK, and prepared as instructed by the manufacturer. Aliquots were stored at $-80^{\circ} \mathrm{C}$ until required. Samples of human albumin (Sigma Aldrich, $10 \mathrm{wt} \%$ ), and $\mathrm{CaCl}_{2}$ (Fluka, $1 \mathrm{M}$ ) were stored at $4^{\circ} \mathrm{C}$ until required. Samples of fibrinogen and thrombin were allowed to thaw at room temperature before being placed on ice. Appropriate amounts of albumin diluted to $4.5 \mathrm{wt} \%$ in Tris Buffered Saline (Sigma Aldrich), fibrinogen and $\mathrm{CaCl}_{2}$ were mixed (in that order) to give the required final concentration of $c=10 \mathrm{mg} / \mathrm{ml}$ fibrinogen and $0.005 \mathrm{M} \mathrm{CaCl}_{2}$. Thrombin was added to initiate gelation at a final concentration $\varphi=0.07 \mathrm{NIH} / \mathrm{ml}$ immediately prior to the sample's transfer to a custom made lower plate mounted on the temperature-controlled stage of the rheometer.

The plate incorporated a glass cover slip providing an optical path through the sample. The stage and plate temperature was maintained at $10^{\circ} \mathrm{C}$. A thin $(\sim 10 \mu \mathrm{m})$ polyethylene film secured on the upper Cone $\left(60 \mathrm{~mm}\right.$ diameter, $2^{\circ}$ cone angle) provided a defect free, readily removed cover over the surface of the rheometer measuring geometry. The sample's outer free surface was covered with a thin layer of low viscosity silicon oil to minimise evaporative losses. A series of CSPS measurements were conducted on fibrinogen-thrombin gels (all $c=10 \mathrm{mg} / \mathrm{ml}, \varphi=0.07 \mathrm{NIH} / \mathrm{ml}$ ) and the steady applied stress $\sigma_{s}$ used to generate the unidirectional flow was adjusted in each experiment.

\subsection{Laser Scanning Confocal Microscopy, LSCM}

Following the attainment of a GP in the experiments described in Section 2.4, the rheometer geometry was locked in position and a scalpel was used to release and fold back the polyethylene film. The geometry was subsequently raised to the loading position, leaving the fibrin gel covered by the film. The aluminium plate was immediately mounted on the stage of a Zeiss LSM 710 inverted confocal microscope. A $63 \times$ (1.4 N.A.) oil immersion objective was focused through the cover slip built into the aluminium plate to a distance of approximately $20 \mu \mathrm{m}$ into the fibrin gel. Using a $488 \mathrm{~nm}$ Argon ion laser and the microscope set in reflection mode, a stack of images measuring $135 \mu \mathrm{m}(\mathrm{W}) \times 135 \mu \mathrm{m}(\mathrm{H}) \times 40 \mu \mathrm{m}$ (D) was recorded.

\subsection{Scanning Electron Microscopy, SEM}

After recording LSCM images, the same sample used in CSPS and LSCM was transferred to a Petri dish and washed three times with $50 \mathrm{mM}$ Sodium Cacodylate- $\mathrm{HCl}$ Buffer solution (pH 7.2-7.4, SPI Supplies) at 10 to 20 minute intervals to remove excess salt. The gel was fixed overnight in $2 \%$ Glutaraldehyde (Sigma Aldrich, UK) and the sample was dehydrated with a series of graded concentrations (30\% to $100 \%$ ) of ethanol. The dehydrated sample was then rinsed with 50\% Hexamethyldisilazane solution 
(HMDS) in 100\% ethanol for 10 minutes in a fume hood and then three times in 100\% HMDS and left overnight to dry. The sample was coated with a thin layer of gold $(\sim 15 \mathrm{~nm})$ using sputter coating and was imaged using scanning electron microscopy (Hitachi 4800).

\section{Results}

For incipient blood clots the value of $d_{f}(1.75)$ in SAOS $\left(\sigma_{s}=0 \mathrm{~Pa}\right)$ is within the Healthy Index range previously reported [14]. As $\sigma_{s}$ is increased progressively in a series of CSPS tests, the value of $d_{f}$ increases from $d_{f}=1.75$ to a maximum value $\left(d_{f}=2.20\right)$ at $\sigma_{s}=0.141 \mathrm{~Pa}$. Further increases in $\sigma_{s}$, to $0.235 \mathrm{~Pa}$, produced no further significant increase in $d_{f}$ and no evidence of incipient clot formation could be detected (no GP was recorded) for $\sigma_{s}>0.235 \mathrm{~Pa}$. The values of the incipient clot formation time, $T_{G P}$, under CSPS increased from $133( \pm 37)$ seconds (at $\sigma_{s}=0 \mathrm{~Pa}$ ) to $182( \pm 43)$ seconds at $\sigma_{s}=0.141 \mathrm{~Pa}$. No further increase in $T_{G P}$ was recorded for stresses in the range $0.141<\sigma_{s}<0.235 \mathrm{~Pa}$ (see Fig. 2). An example of the CSPS results obtained for blood is shown in Fig. 3 whilst Fig. 4 shows the power law scaling of $G^{\prime}$ and $G^{\prime \prime}$ for a fibrin thrombin gel under SAOS.

Figure 5 shows the relationship between $d_{f}$ and levels of incipient clot elasticity, $G_{G P}^{\prime}$, normalized by their respective values at $d_{f}=2.0, G_{G P}^{\prime}$ being a mechanical property relevant to the clot's haemostatic function. The values of $d_{f}$ and $G_{G P}^{\prime}$ for SAOS of fibrin gels over a range of thrombin concentration $(\varphi=0.01$ to $0.19 \mathrm{NIH} / \mathrm{ml})$ were obtained from a previous study [12]. A striking feature of the 'structurefunction' relationship defined by this plot (a version of which has previously been reported in the polymer literature [34]) is that all the results for blood (in CSPS) and fibrin gels (in SAOS and CSPS tests) can be represented by a single exponential relationship. For fibrin gels under SAOS the increase in $d_{f}$ and $G^{\prime}$ is solely due to gel formation under progressively higher levels of ab initio thrombin concentration, with $d_{f}=2.0$ being the upper limiting value. For blood and fibrin gels under CSPS the higher values of $d_{f}$ and $G_{\| \mathrm{GP}}^{\prime}$ reflect clot formation under unidirectional flow.

The LSCM images shown in Fig. 6 were recorded immediately following attainment of the GP and illustrate the striking effects of unidirectional shear flow on fibrin clot microstructure. The fibrin network
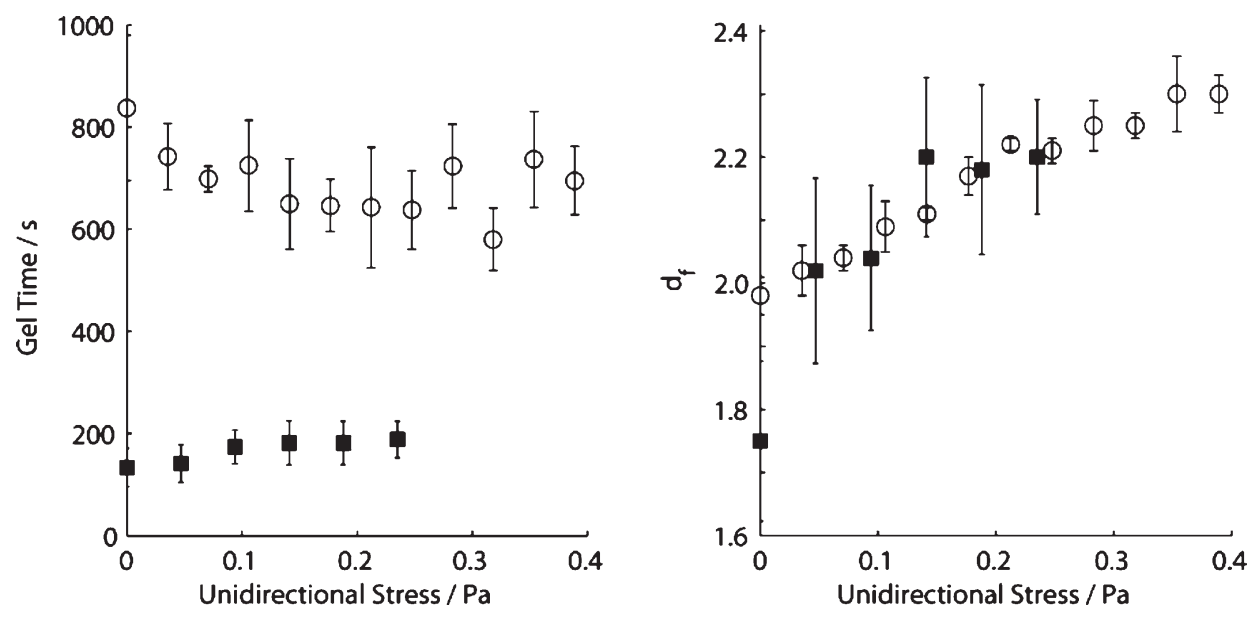

Fig. 2. Variation of gel time (left) and fractal dimension (right) for increasing levels of unidirectional shear stress in blood (squares) and fibrin thrombin gels (circles). 


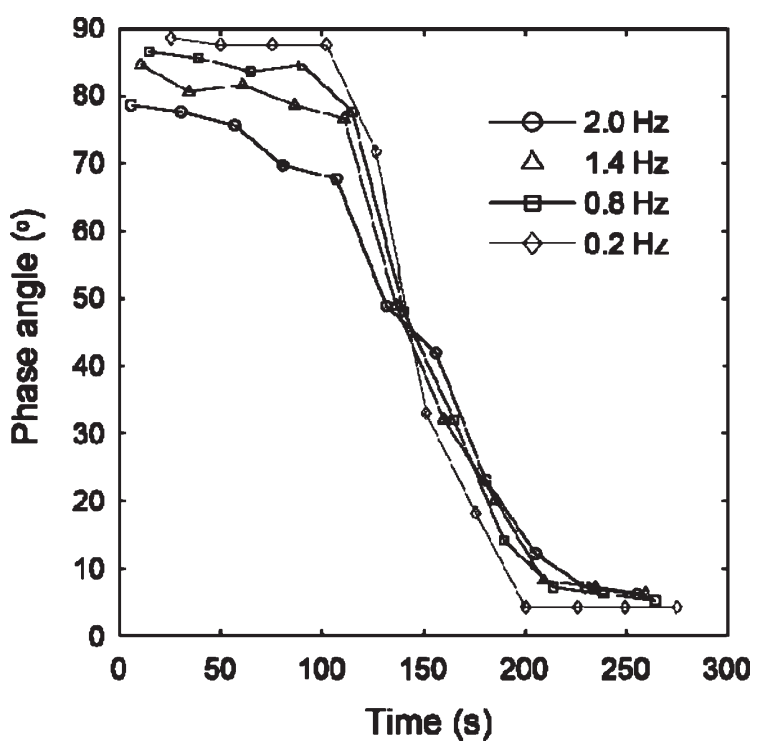

Fig. 3. CSPS results for whole blood. The unidirectional shear stress is $\sigma_{s}=0.047 \mathrm{~Pa}$. The frequency independent value of $\delta$ marks the GP and the establishment of an incipient clot characterized by $d_{f}=2.0$, significantly higher than that recorded under SAOS $\left(d_{f}=1.75\right)$. The maximum value of the unidirectional shear rate $\dot{\gamma}_{s}\left(4 \mathrm{~s}^{-1}\right)$ was recorded at the beginning of the test, $\dot{\gamma}_{s}$ decreasing progressively due to rheological change as the GP is approached. The value of $\dot{\gamma}_{s}$ immediately prior to the GP was $0.1 \mathrm{~s}^{-1}$, becoming vanishingly small thereafter.

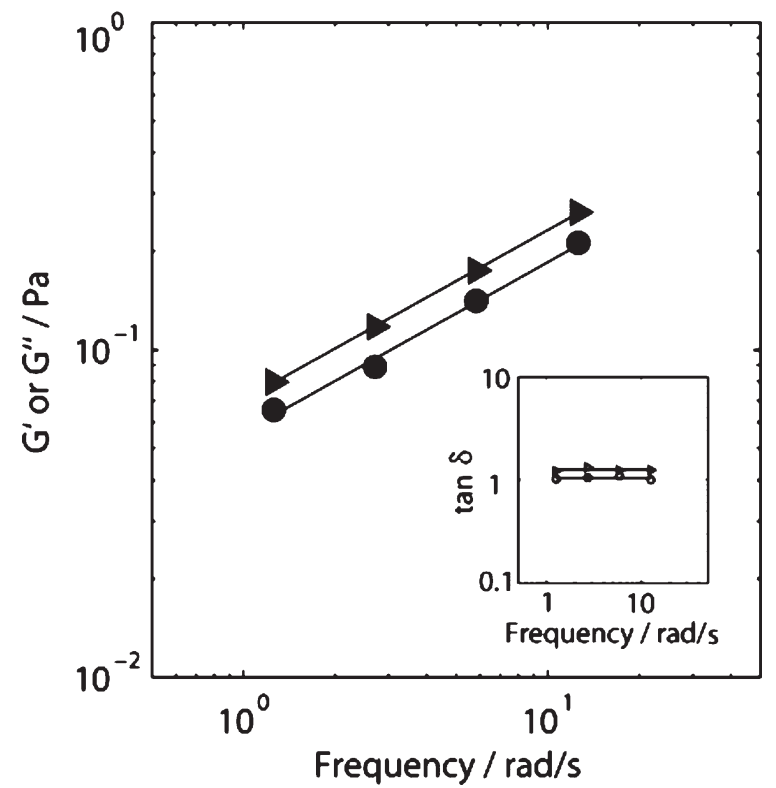

Fig. 4. Values of elastic modulus, $G^{\prime}$ (circles) and loss modulus $G^{\prime \prime}$ (triangles) obtained as a function of angular frequency in SAOS tests on a fibrin-thrombin gel at the GP. Inset shows the corresponding frequency independent values of $\tan \delta\left(=G^{\prime \prime} / G^{\prime}\right)$ for a fibrin gel formed under SAOS (open triangles) and CSPS with $\sigma=0.177 \mathrm{~Pa}$ (open circles). The results reveal a decrease in $\tan \delta$ (corresponding to an increase in $d_{f}$ ) under CSPS relative to SAOS. 


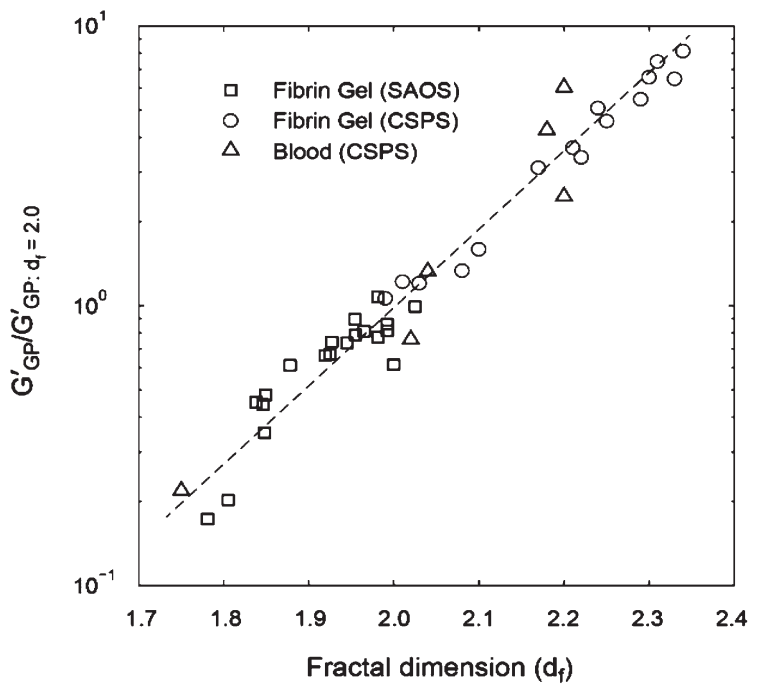

Fig. 5. Structure-function relationship in terms of $d_{f}$ and incipient clot elasticity for whole blood (CSPS) and fibrin gels (in SAOS and CSPS tests). The values of $G_{G P}^{\prime}$ and $G_{\| G P}^{\prime}$ are normalized by their respective values at $d_{f}=2.0$. The SAOS results for fibrin gels result from a progressive increase in thrombin concentration at a fixed value of fibrinogen concentration $[\varphi=0.01$ to $0.19 \mathrm{NIH} / \mathrm{ml}, c=10 \mathrm{mg} / \mathrm{ml}$.

structure incorporates progressively more mass as the unidirectional flow stress $\sigma_{s}$ increases, a finding consistent with the elevated values of $\mathrm{d}_{\mathrm{f}}$ found in CSPS.

The scanning electron micrographs (SEMs) (see Fig. 7) obtained for the same samples used in confocal imaging and rheology tests reveal that, the distribution of fibrin fibre diameter increases as the shear stress increases. The fibre diameter distribution (see Fig. 8) was analysed using Image J (V 510, National Institutes of Health) by placing a random grid of crosses (500 crosses/image) on images and using the line tool to measure fibre diameter. Networks formed at $\sigma_{s}=0 \mathrm{~Pa}$ (i.e. under SAOS) exhibited the characteristic homogenous/quiescent gel. In contrast, networks formed under different levels of superposed shear stress $\left(\sigma_{s}=0.1,0.17,0.35 \mathrm{~Pa}\right)$ showed a prominent network of thick fibres, as well as fibre 'aggregates' in which multiple individual fibres were coalesced into bundles, several of which had diameters greater than $1 \mu \mathrm{m}$. These changes could be the result of physical forces of stress on the fibre growth or the transport of oligomers as a result of the stress.

\section{Discussion}

The unidirectional shear stresses employed in this CSPS study are lower than those previously reported to influence the elasticity of fully formed clots in SAOS tests following exposure of coagulating plasma to flow [18]. A significant aspect of the CSPS results is that they reveal the marked sensitivity of incipient blood clot microstructure $\left(d_{f}\right)$ to unidirectional flow shear stresses $<0.3 \mathrm{~Pa}$. Such low stresses have physiological relevance insofar as they are commensurate with estimates of the mean wall shear stress for flow in the aorta, inferior vena cava and other venous structures $[6,21]$. The potential clinical significance of the increase in $d_{f}$ under CSPS is that fractal networks such as the incipient clot require large amounts of additional mass to produce small increments in $d_{f}$. Further, given the incipient clot's role 

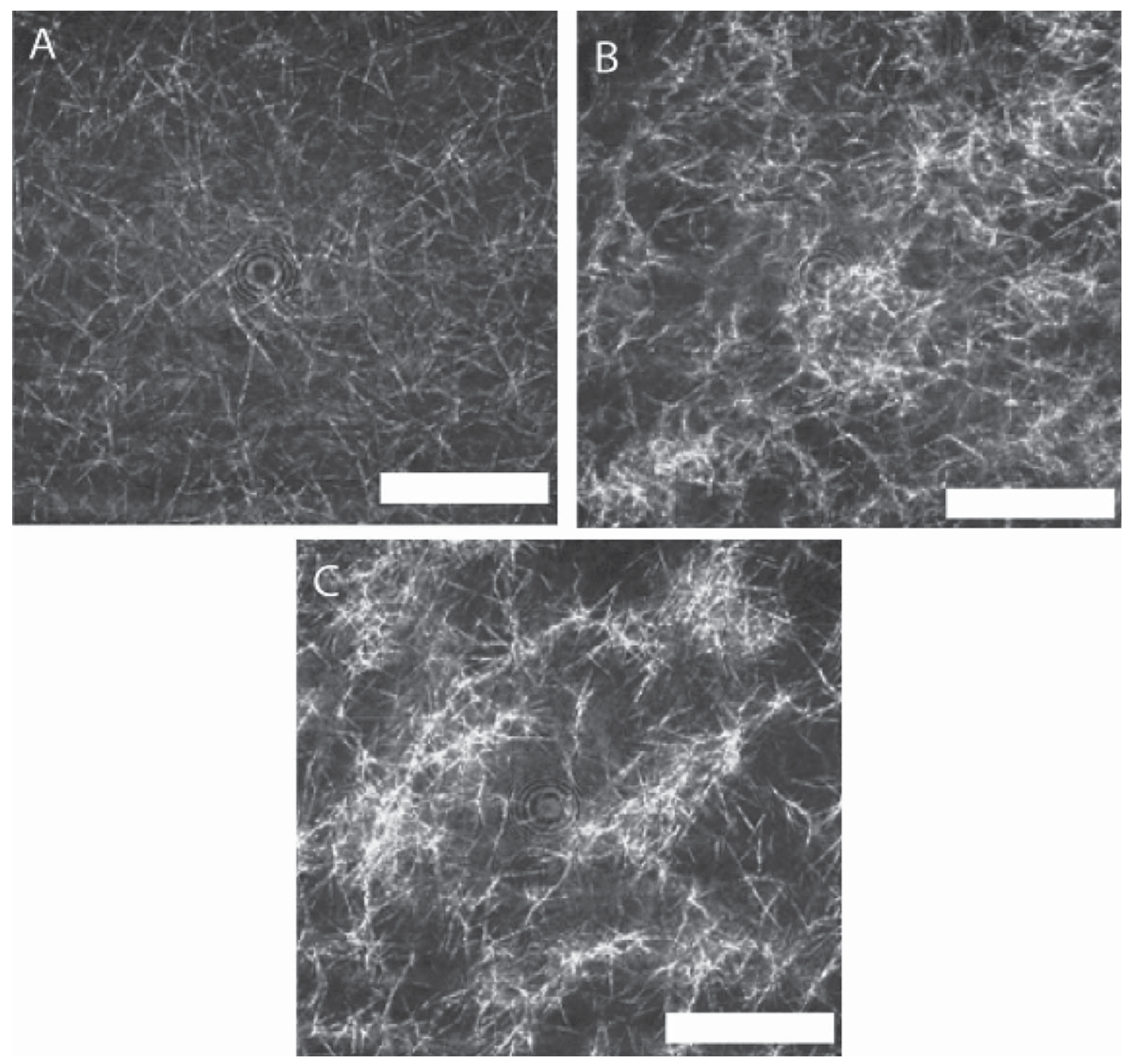

Fig. 6. LSCM images of a fibrin gel formed under SAOS and those formed under CSPS at three different levels of shear stress. Fibrin gel network formed under (A) SAOS ( $\left.\sigma_{s}=0 \mathrm{~Pa}\right),(\mathrm{B}) \operatorname{CSPS}\left(\sigma_{s}=0.1 \mathrm{~Pa}\right)$ and (C) CSPS $\left(\sigma_{s}=0.35 \mathrm{~Pa}\right)$ with the corresponding values of $d_{f}$ being $1.99( \pm 0.01), 2.03( \pm 0.01)$ and $2.3( \pm 0.05)$, respectively. The images were acquired immediately following the attainment of the GP in the rheometer. The scale bar width is $20 \mu \mathrm{m}$.

as a microstructural template for ensuing clot development [12], elevated values of $d_{f}$ might lead to the establishment of denser, less permeable clots with enhanced resistance to fibrinolysis [8-10] and deformation [32]. The latter characteristic is commensurate with the present results in terms of the significant correlation between $d_{f}$ and shear elastic modulus, $G_{\mathrm{GP}}^{\prime}$ and $G_{\| \mathrm{GP}}^{\prime}$.

A striking finding is that all the results for $d_{f}$ and normalized elasticity for blood (under CSPS) and fibrin gels (under SAOS and CSPS) are represented by a single exponential relationship (see Fig. 5) - a feature consistent with the underlying fractal nature of the fibrin network assembly. Despite the presence of cellular material in whole blood, the relationship between clot microstructure $\left(d_{f}\right)$ and elasticity is the 

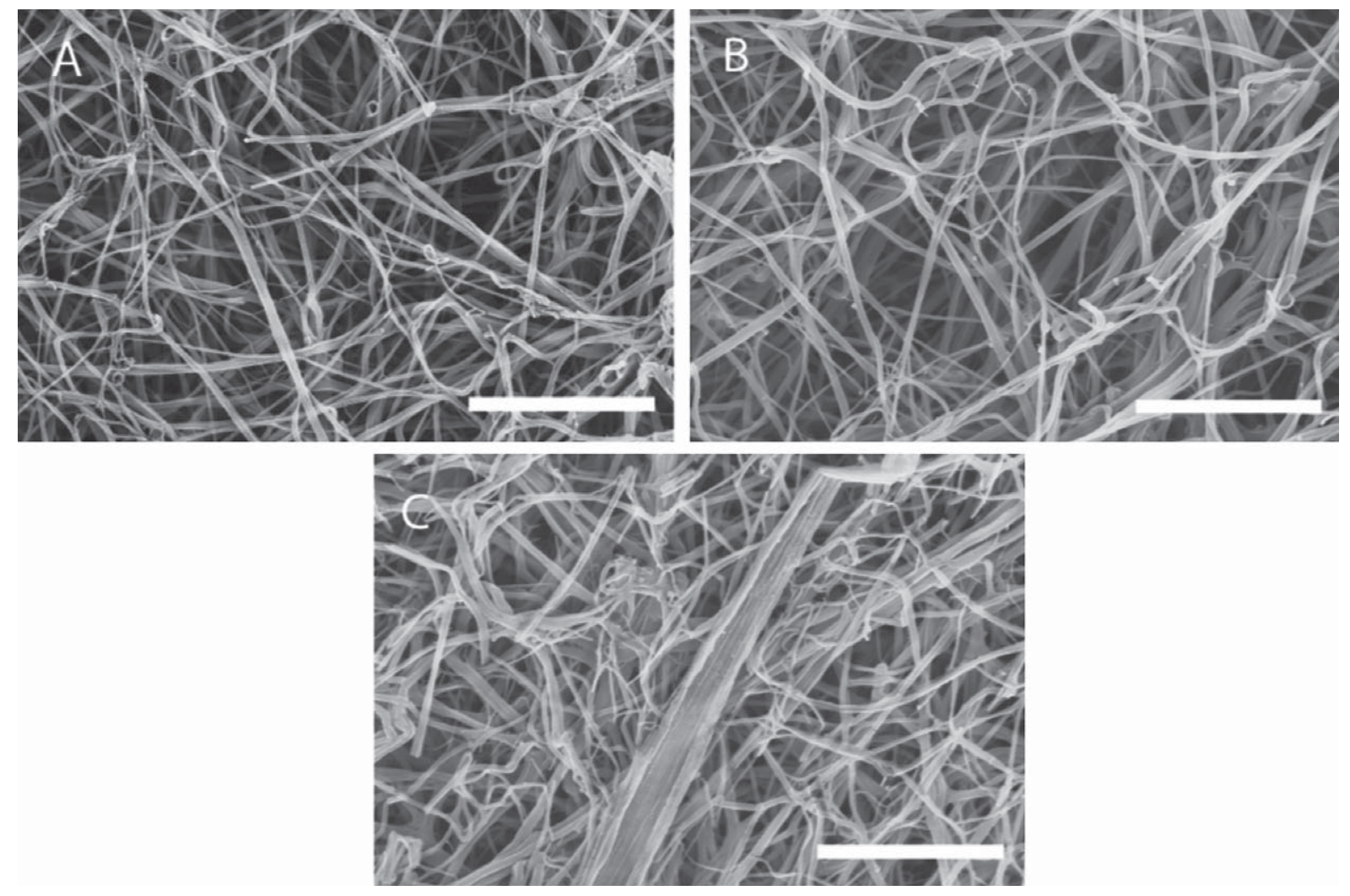

Fig. 7. SEM images of a fibrin gel formed under SAOS and those formed under CSPS at different levels of shear stress. Fibrin gel network formed under (A) SAOS $\left(\sigma_{s}=0 \mathrm{~Pa}\right)$, (B) CSPS $\left(\sigma_{s}=0.1 \mathrm{~Pa}\right)$ and (C) CSPS $\left(\sigma_{s}=0.35 \mathrm{~Pa}\right)$ with the corresponding values of $d_{f}$ being $1.99( \pm 0.01), 2.03$ ( \pm 0.01$)$ and 2.3 ( \pm 0.05 ), respectively. The scale bar width is $5 \mu \mathrm{m}$.

same as that found in fibrin clots - systems which have no other microstructural component. Thus, in terms of this 'structure-function' relationship, the present results confirm the fibrin network as the dominant microstructural component of the incipient clot in terms of determining its response to imposed stress.

The incorporation of greater mass within the fibrin network under flow would be expected to result in increased elasticity as the shear elastic modulus correlates with branchpoint density and elevated values of the latter correspond to higher values of $d_{f}$ [32]. High levels of thrombin are associated with the production of thin fibrin fibres with many branchpoints and enhanced monomer activation being expected to result from more effective mixing of the available thrombin under flow $[1,26]$. Molecular dynamic simulations (MDS) of incipient fractal clot formation, based on the activation-limited aggregation of rodlike particles into sample-spanning GP clusters, reveal that enhanced monomer activation, generated by activation profiles representing differing rates of thrombin production, increases (i) the rate of addition of mass inside the growing molecular clusters; and (ii) the diffusion and transport of mass in the intra-cluster voids [11]. Hence, under the enhanced monomer activation resulting from mixing in shear flow, clots form from smaller units with relatively large amounts of incorporated mass, and correspondingly high $d_{f}$. This suggests that the increase in $d_{f}$ in blood may be attributable to shear enhanced thrombin generation.

A previous SAOS study of fibrin gel formation reported that at high (ab initio) thrombin concentration $d_{f}$ reached a limiting maximum value (ca. $d_{f} \sim 2.0$ ) [12]. Under CSPS, at the same values of $\mathrm{c}$ and $\varphi$, 

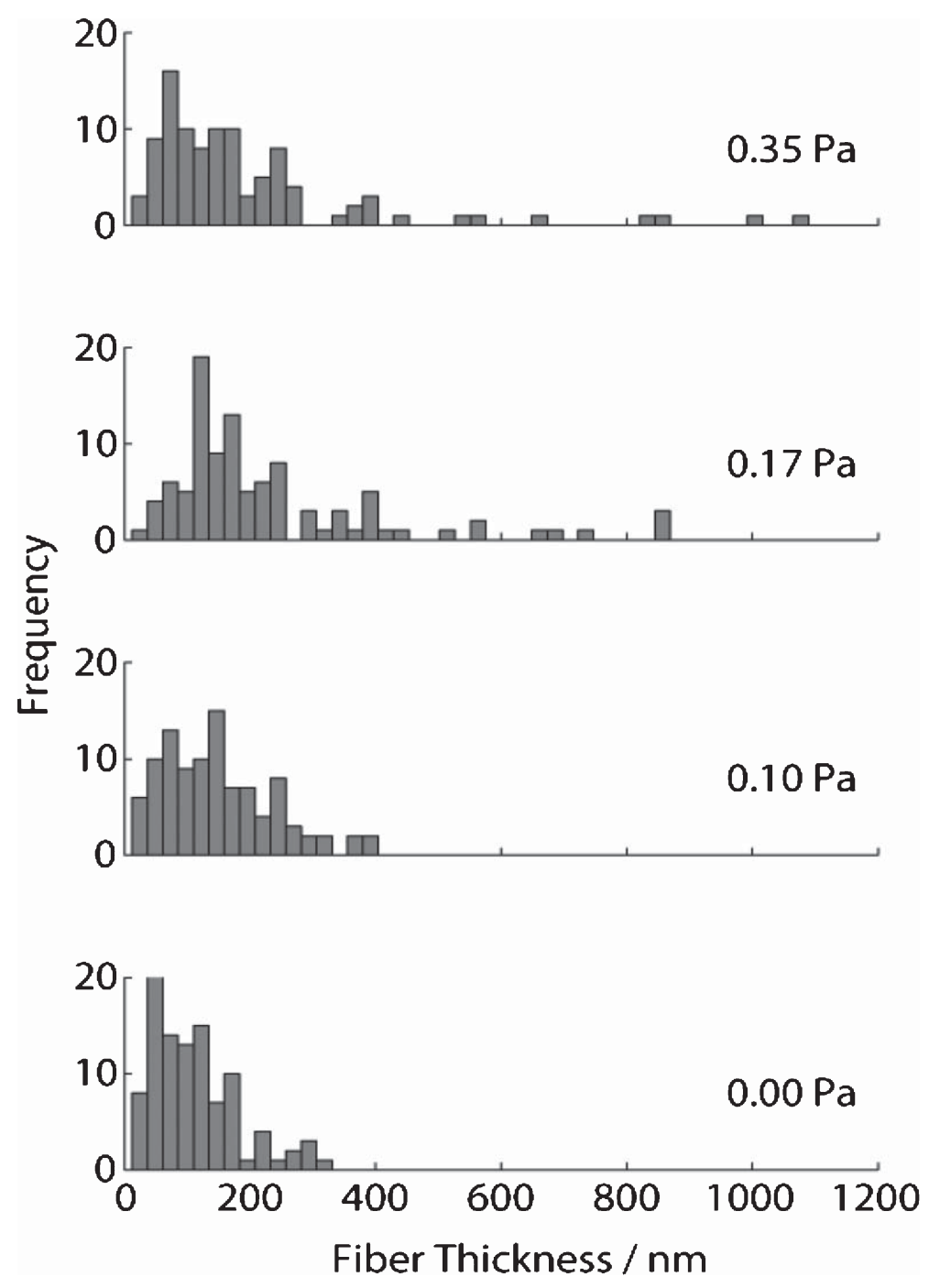

Fig. 8. Measurements of fibrin fibre diameter from analysis of SEM images of clots formed under SAOS (lower, $\left.\sigma_{s}=0 \mathrm{~Pa}\right)$ and different levels of applied unidirectional shear stress in CSPS.

the value of $d_{f}$ increases progressively in the range $2.0<d_{f}<2.3$ with increasing stress $\sigma_{s}$. These findings indicate that in the absence of the mixing/activation effect of a unidirectional shear flow, $a b$ initio thrombin addition cannot alone induce sufficient activation to produce the substantial additional fibrin mass required to achieve the highest values of $d_{f}(>2.0)$ recorded in CSPS.

Following an initial increase in incipient clot formation time $T_{G P}$ in coagulating blood under CSPS at low stresses, no further increase was observed in the range $0.141<\sigma_{s}<0.235 \mathrm{~Pa}$. This may be understood in terms of the disruptive influence of the unidirectional flow on the growing pre-GP clusters as the accumulated strain increases during clotting. Carvalho and Djabourov [4] have reported biopolymer gelation under alternating sequences of oscillatory flow and steady shear flow in which the flow kinematics caused gel disruption. In the present CSPS work on blood, the unidirectional flow produces two competing 
effects. While the shear field serves to delay the GP, enhanced platelet activation would eventually counterbalance this effect to produce the constant value of $\mathrm{T}_{\mathrm{GP}}$ and result in a higher value of $d_{f}$ when the incipient clot eventually forms (i.e. when the competing effect of pre-GP cluster growth is sufficient to establish a sample-spanning network). The maximum unidirectional shear rates in the CSPS experiments were typically less than $10 \mathrm{~s}^{-1}$, the increase in $d_{f}$ recorded under these conditions being consistent with observations by Campbell et al. [3] who note that shear flow modulates the kinetics of both fibrin monomer formation and polymerisation. Ultimately, at sufficiently high stress, no GP is recorded under CSPS, a finding commensurate with a reported decrease in platelet-fibrin interaction with increasing shear stress exposure at sufficiently high levels of stress in SAOS studies [18]. Given that there is no (permanent) fibrin fibre orientation under SAOS, it is interesting to consider whether the elevated values of $d_{f}$ may be attributable to accumulated fibrin fibre orientation under the unidirectional flow component of CSPS. The LSCM and SEM images do not suggest significant fibrin fibre orientation in the CSPS results due, presumably, to the low levels of shear stress involved in the CSPS experiments and the low levels of shear elasticity associated with the incipient clot. However the results provide evidence of enhanced fibre bundling under flow, a feature which has been reported for mature clot microstructures [3, 17, 27].

\section{Conclusions}

The results of this first study of blood coagulation by CSPS suggest that it is a valuable haemorheological tool in the study of modified clot microstructure. They also lend further support to the hypothesis that $d_{f}$ predominantly reflects the fibrin network arrangement, and its role as the principal microstructural component of the incipient clot [14]. The results illustrate the relationship between this microstructural parameter and a mechanical property (shear elasticity) underlying clot haemostatic functionality. Moreover, the elevation of $d_{f}$ above the values previously attained in fibrin clots by increasing thrombin concentration is evidence of the ability of unidirectional flow to enhance activation and clot assembly, leading to the incorporation of more polymerized mass within the incipient clot. This feature, along with the corresponding elevation of incipient clot elasticity, may have clinical significance in terms of modified clot structure in disease states [1]. Instances of incipient clot formation featuring substantially enhanced mass and elasticity clearly merit further study. Since even the relatively low shear rates studied here have significant effects on clot microstructure and mechanical properties, the much greater shear rates encountered in stenotic vessels associated with thrombosis could have even more striking effects.

\section{Acknowledgments}

This work was supported by EPSRC(UK) grants EP/C513037/ 1 and EP/I019405/1 and a NISCHR BRU award.

\section{References}

[1] B. Blomback and N. Bark, Fibrinopeptides and fibrin gel structure, Biophys Chemist 112 (2004), 147-151.

[2] H.C. Booij, Influence of superimposed steady shear flow on the dynamic properties on non-Newtonian fluids I: Measurements on non-Newtonian solutions, Rheol Acta 5 (1966), 215-221.

[3] R.A. Campbell, M. Aleman, L.D. Gray, M.R. Falvo and A.S. Wolberg, Flow profoundly influences fibrin network structure: Implications for fibrin formation and clot stability in haemostasis, Thromb Haemost 104 (2010), 1281-1284. 
[4] W. Carvalho and M. Djabourov, Physical gelation under shear for gelatin gels, Rheol Acta 36 (1997) 591-609.

[5] F. Chambon and H.H. Winter, Linear viscoelasticity at the gel point of a crosslinking PDMS with imbalanced stoichiometry, J Rheol 31 (1987), 683-697.

[6] C.P. Cheng, R.J. Herfkens and C.A. Taylor, Inferior vena caval hemodynamics quantified in vivo at rest and during cycling exercise using magnetic resonance imaging, Am J Physiol Heart Circ Physiol 284 (2003), H1161-H1167.

[7] T.V. Colace, R.W. Muthard and S.L. Diamond, Thrombus growth and embolism on tissue factor-bearing collagen surfaces under flow role of thrombin with and without fibrin, Arterioscler Thromb Vasc Biol 32 (2012), 1466-1476.

[8] J.P. Collet, Y. Allali, C. Lesty, M.L. Tanguy, J. Silvain, et al., Altered fibrin architecture is associated with hypofibrinolysis and premature coronary atherothrombosis, Arterioscler Thromb Vasc Biol 26 (2006), 2567-2573.

[9] J.P. Collet, C. Lesty, G. Montalescot and J.W. Weisel, Dynamic changes of fibrin architecture during fibrin formation and intrinsic fibrinolysis of fibrin-rich clots, J Biol Chem 278 (2003), 21331-21335.

[10] J.P. Collet, D. Park, C. Lesty, J. Soria, C. Soria, G. Montalescot, et al., Influence of fibrin network conformation and fibrin fiber diameter on fibrinolysis speed: Dynamic and structural approaches by confocal microscopy, Arterioscler Thromb Vasc Biol 20 (2000), 1354-1361.

[11] D.J. Curtis, M.R. Brown, K. Hawkins, P.A. Evans, M.J. Lawrence, P. Rees and P.R. Williams, Rheometrical and molecular dynamics simulation studies of incipient clot formation in fibrin-thrombin gels: An activation limited aggregation approach, J Non-Newton Fluid Mechanics 166 (2011), 932-938.

[12] D.J. Curtis, P.R. Williams, N. Badiei, A.I. Campbell, K. Hawkins, P.A. Evans and M.R. Brown, A study of microstructural templating in fibrin-thrombin gel networks by spectral and viscoelastic analysis, Soft Matter 9 (2013), 4883-4889.

[13] P.A. Evans, K. Hawkins, M. Lawrence, R.L. Williams, M.S. Barrow, N. Thirumalai and P.R. Williams, Rheometry and associated techniques for blood coagulation studies, Med Eng Phys 30 (2008), 671-679.

[14] P.A. Evans, K. Hawkins, R.H.K. Morris, N. Thirumalai, R. Munro, L. Wakeman, et al., Gel point and fractal microstructure of incipient blood clots are significant new markers of hemostasis for healthy and anticoagulated blood, Blood 116 (2010), $3341-3346$

[15] P.A. Evans, K. Hawkins, P.R. Williams and R.L. Williams, A study of gelatin gelation by Fourier transform mechanical spectroscopy, J Non-Newtonian Fluid Mechanics 148 (2008), 122-126.

[16] F. Ferri, M. Greco, G. Arcovito, M. De Spirito and M. Rocco, Structure of fibrin gels studied by elastic light scattering techniques: Dependence of fractal dimension, gel crossover length, fiber diameter, and fiber density on monomer concentration, Phys, Rev E 66 (2002), 011913.

[17] K.C. Gersh, K.E. Edmondson and J.W. Weisel, Flow rate and fibrin fiber alignment, J Thromb Haemost 8 (2010), $2826-2828$.

[18] C.J. Glover, L.V. McIntire, L.B. Leverett, J.D. Hellums, C.H. Brown and E.A. Natelson, Effect of shear stress on clot structure formation, ASAIO Journal 20 (1974), 463-468.

[19] K. Hawkins, P.A. Evans, M. Lawrence, D. Curtis, T.M. Davies and P.R. Williams, The development of rheometry for strain-sensitive gelling systems and its application in a study of fibrin-thrombin gel formation, Rheol Acta 49 (2010), 891-900.

[20] F. Jung, S. Braune and A. Lendlein, Haemocompatibility testing of biomaterials using human platelets, Clin Hemorheol Microcirc 53 (2013), 97-115.

[21] M.H. Kroll, J.D. Hellums, L.V. Mclntire, A. Schafer and J.L. Moake, Platelets and shear stress, Blood 88 (1996), 1525-1541.

[22] M.J. Lawrence, S. Kumar, K. Hawkins, S. Bodend, H. Rutt, G. Mills, A. Sabra, R.H.K. Morris, S.J. Davidson, N. Badiei, M.R. Brown, P.R. Williams and P.A. Evans, A new structural biomarker that quantifies and predicts changes in clot strength and quality in a model of progressive haemodilution, Thrombosis Research 134 (2014), 488-494.

[23] M.J. Lawrence, A. Sabra, G. Mills, S.G. Pillai, W. Abdullah, K. Hawkins, R.H.K. Morris, S.J. Davidson, L.A. D’Silva, D.J. Curtis, M.R.Brown, J.W. Wiesel, P.R. Williams and P.A. Evans, A new biomarker quantifies differences in clot microstructure in patients with venous thromboembolism, British Journal of Haematology (2014). DOI: 10.1111/bjh.13173

[24] J.D. Mills, R.A. Ariens, M.W. Mansfield and P.J. Grant, Altered fibrin clot structure in the healthy relatives of patients with premature coronary artery disease, Circulation 106 (2002), 1938-1942.

[25] D.M. Monroe, M. Hoffman and H.R. Roberts, Platelets and thrombin generation, Arterioscler Thromb Vasc Biol 22 (2002), 1381-1389.

[26] M. Mosesson, Fibrinogen and fibrin structure and functions, J Thrombosis and Haemostasis 3 (2005), 1894-1904.

[27] S. Muenster, L.M. Jawert, B. Fabry and D.A. Weitz, Structure and mechanics of fibrin clots formed under mechanical perturbation, J Thromb Haemost 11 (2013), 557-560.

[28] M. Muthukumar, Screening effect on viscoelasticity near the gel point, Macromolecules 22 (1989), 4656-4658. 
[29] K.B. Neeves, D.A. Illing and S.L. Diamond, Thrombin flux and wall shear rate regulate fibrin fiber deposition state during polymerization under flow, Biophys $J 98$ (2010), 1344-1352.

[30] I.K. Piechocka, R.G. Bacabac, M. Potters, F.C. MacKintosh and G.H. Koenderink, Structural hierarchy governs fibrin gel mechanics, Biophysical Journal 98 (2010), 2281-2289.

[31] W.H. Reinhart, Platelets in vascular disease, Clin Hemorheol Microcirc 53 (2013), 71-79.

[32] E.A. Ryan, L.F. Mockros, J.W. Weisel and L. Lorand, Structural origins of fibrin clot rheology, Biophys J 77 (1999), 2813-2826.

[33] L.M. Sander, Fractal growth processes, Nature 322 (1986), 789-793.

[34] J.C. Scanlan and H.H. Winter, Composition dependence of the viscoelasticity of end-linked poly(dimthylsiloxane) at the Gel Point, Macromolecules 24 (1991), 47-54.

[35] E. Somma, O. Valentino, G. Titomanlio and G. Ianniruberto, Parallel superposition in entangled polydisperse polymer melts: Experiments and theory, J Rheol 51 (2007), 987.

[36] A. Undas and R.A. Ariens, Fibrin clot structure and function: A role in the pathophysiology of arterial and venous thromboembolic diseases, Arterioscler Thromb Vasc Biol 31 (2011), 88-99.

[37] I. Varju, P. Sotonyi, R. Machovich, L. Szabo, K. Tenekedjiev, M.M.C.G. Silva, et al., Hindered dissolution of fibrin formed under mechanical stress, J Thromb Haemost 9 (2011), 979-986.

[38] J. Weisel, The mechanical properties of fibrin for basic scientists and clinicians, Biophys Chemist 112 (2004), 267-276.

[39] J.W. Weisel and R.I. Litvinov, Mechanisms of fibrin polymerization and clinical implications, Blood 11 (2013), $1712-1719$.

[40] D.A. Weitz and M. Oliveria, Fractal structures formed by kinetic aggregation of aqueous gold colloids, Physical Review Letters 52 (1984), 1433.

[41] P. Whittaker and K. Przyklenk, Fibrin architecture in clots: A quantitative polarized light microscopy analysis, Blood Cells Mol Dis 42 (2009), 51-56.

[42] D. Young and E. Bermes, Specimen collection and processing sources of biological variation, in Tietz textbook of clinical chemistry Part 3 ed C.A. Burtis and E.R. Ashwood, WB Saunders, Philadelphia, 1999, pp. 42-72. 